\title{
Author Correction: Rare genetic variants affecting urine metabolite levels link population variation to inborn errors of metabolism
}

\author{
Yurong Cheng, Pascal Schlosser $\mathbb{D}^{0}$, Johannes Hertel, Peggy Sekula $\mathbb{D}^{0}$, Peter J. Oefner, Ute Spiekerkoetter, \\ Johanna Mielke, Daniel F. Freitag (1), Miriam Schmidts (1) \& GCKD Investigators ${ }^{\star}$, Florian Kronenberg (1D, \\ Kai-Uwe Eckardt (1), Ines Thiele, Yong Li (1) \& Anna Köttgen (10)
}

Correction to: Nature Communications https://doi.org/10.1038/s41467-020-20877-8, published online 11 February 2021.

The original version of the Supplementary Data associated with this Article included two incorrect column labels in Supplementary Data 3 and 6 files. All numeric content remains unchanged. The HTML has been updated to include a corrected version of Supplementary Data 3 and 6; the original version of Supplementary Data 3 and 6 with the incorrectly labelled column headers can be found as Supplementary Information associated with this Correction.

Published online: 06 October 2021

\section{Additional information}

Supplementary information The online version contains supplementary material available at https://doi.org/10.1038/s41467-021-26242-7. 